ABSTRACT ID: 90

Poster(Competing)

\title{
The Antimicrobial Effect Of Virgin Coconut Oil On Oral Pathogen
}

Nur Ainatul Mardia Bt Mohamad Nasir ${ }^{a}$ | Zurainie Bt Abllah ${ }^{b}$ | Intan Azura Bt Shahdan ${ }^{c}$ | Anil Azura Bt Jalaludin ${ }^{a}$

${ }^{a}$ Department of Biotechnology, Kulliyyah of Science, IIUM I ${ }^{b}$ Department of Paediatric, Orthodontic \& Dental Public Health, Kulliyyah of Dentistry, IIUM| 'Department of Biomedical Science, Kulliyyah of Allied Health Science, IIUM

Introduction: As time progressed, our diet changed to include the types of foods that will promote decay and so dental caries became a widespread and serious concern. Dental caries had been reported adding to the significantly high number of oral health problem worldwide, causing a burden to health care system. Despite the existing of prevention courses, the numbers did not appear to decrease. Originated from natural resources, virgin coconut oil (VCO) has been proposed as an alternative treatment as it has been reported to demonstrate antimicrobial activity on various bacteria. Hence, the present study aimed to investigate whether VCO has antimicrobial effects on certain, selected cariogenic bacteria. Materials and Methods: Crude extract of VCO was prepared by using a natural method of fermentation. The composition of VCO were screened using Gas Chromatography Mass Spectrophotometry (GCMS). The antimicrobial activities of VCO were evaluated against three selected oral pathogens; Streptococcus mutans, Lactobacillus casei and Candida albicans using disc diffusion method. The inhibitory activities of VCO were determined by measuring the diameter of inhibition zone. The antimicrobial activities were further tested using broth micro-dilution technique to determine the minimum inhibitory concentration (MIC) and minimum bactericidal concentration (MBC). Results: The inhibition zone was observed on Candida albicans plate, however no inhibitions were observed on Streptococcus mutans and Lactobacillus casei plates. The MIC and MBC results were inconsistent in 3 duplication that have been done for all the bacteria. Conclusion(s): VCO demonstrated potential antimicrobial activity against Candida albicans but not on Streptococcus mutans and Lactbacillus casei.

KEYWORDS: virgin coconut oil, dental caries, candida infection 